\title{
Low-vacuum Thermal Insulation Panels based on Silicon Dioxide Powders
}

\author{
Rashit Nazirov ${ }^{1}$, Ivan Inzhutov ${ }^{1}$, Alexey Zhzhonykh ${ }^{1}$ and Nikita Novikov ${ }^{1}$ \\ ${ }^{1}$ School of Engineering and Construction, Siberian Federal University, 82 Svobodny, Krasnoyarsk, 660041 Russia
}

\begin{abstract}
The composition and technology of obtaining low-vacuum thermal insulation panels with a powder filler of silicon dioxide and infrared silencer have been developed. The thermal conductivity of the low vacuum insulated products with the use of synthetic silica dioxide and micro-silica - waste production with the addition of titanium dioxide at moderate temperatures with samples of $37.5^{\circ} \mathrm{C}$ and $1.0^{\circ} \mathrm{C}$ is investigated. The results of electron microscopy of materials studied are presented. The optimal amount of additive-infrared silencer is determined. It has been found out that low-vacuum products based on micro-silica with an optimal amount of additives have a thermal conductivity lower than that of the air at lower temperatures.
\end{abstract}

\section{Introduction}

The problem of energy expenditure reducing is topical in connection with regular and very fast energy recourses prices growth. According to the concept of long-term development of the Russian Federation [1], special attention should be paid to innovative research of efficient energy-saving systems for various fields of industry, construction, housing and communal services, etc. Room heat energy loss is because of high coefficient of filler structures thermal conductivity and ventilation. These factors have not been taken into account in construction before because energy carriers were relatively cheap but in the process of being more expensive there was a problem of room energy efficiency. Earlier building heating insulation was formed by wall thickness increasing but at present time there is no need to do it with modern heat insulating materials appearing. These materials are light, frost resistant and they are used not only in new buildings construction but in old ones reconstruction. The modern market offers a wide range of thermal insulation materials for building envelopes, which can significantly reduce the loss of thermal energy during their operation. The use of vacuum insulation panels (VIP) [2-4] seems to be promising keeping in mind the global trend of reducing energy consumption by buildings.

Production of slabs from loose and powder materials by means of vacuuming technology is a promising technology for the manufacture of slab insulation. Vacuuming helps to ensure durability as a result of reducing the negative impact caused by the effect of condensation of water vapor [5]. High - porous materials - poly-disperse granular powders are used as the main component of filling vacuum insulation panels. Physical bases of thermal conductivity of the powders are discussed in details in publications of G. N. Dulnev [6, 7]. Thermal conductivity of dispersed systems is determined by many factors: the thermal conductivity of the solid frame material, the type and convection of gas in the pore space, its pressure, radiation, the porosity of the frame and its individual particles, as well as the thermal conductivity of the contact between these particles $[4,7,8,9,10]$.

The creation of vacuum heat insulating panels is strictly based on laws of Physics proving that pressure absence or decreasing inside porous material increases its heat insulating properties.

The investigated low vacuum insulating panel is produced on the basis of open porous structure filler put in gas resisting casing and has three main components -"Filler" which provides mechanical strength and heat insulating properties by free gas flow (air molecules) preventing and thus decreasing heat transfer ability through air conductivity convective component. Theoretically the basic material must have open cell structure with very small size of pores and infrared radiation;

-"Gas impermeable barrier" which serves as a barrier for air and steam and is a casing for the main material. Vacuum panels heat properties and their durability depend on those characteristics.

-"Infrared jammer" added to the filler to decrease thermal conductivity ray component.

The produced FRONT-VIP vacuum insulation panel of VACU-ISOTEC KG Company (Radeberg, Germany) [11] has a filler in the form of highly dispersed silicon dioxide. As a VIP filler, pyrogenic silica is also used, obtained by flame hydrolysis of high purity tetrachloride silicon [11]. However, the pyrogenic silica has a complex production technology and high cost. Therefore, its 
replacement, without performance degradation, by microsilica is economically and technologically justified.

The TURNA company offers TURVAC $\mathrm{Si}$ тм vacuum panels on the base of aerogel, packed in a lowpermeability multilayer foil, which increases the service life according to the company, to $40 \ldots 60$ years. Low thermal conductivity $(<0.0045 \mathrm{~W} /(\mathrm{m} \cdot \mathrm{K})$ at $20 \mathrm{~mm}$ thickness) is provided by internal pressure of $<5 \mathrm{mbar}$ $(<500 \mathrm{~Pa})$ [12].

VA-Q-tec AG (Würzburg, Germany) offers vacuum insulation panels for va-Q-vip $B$ and va-Q-vip $F$ construction having a thermal conductivity of 0.007 and $0.008 \mathrm{~W} /(\mathrm{m} \cdot \mathrm{K})$ at a thickness of $20 \mathrm{~mm}$ and $10 \ldots 15 \mathrm{~mm}$ respectively. Internal pressure is $<5$ mbar, which may have an increase in 1 mbar per year. The thermal conductivity of the depressurized panel increases to 0.02 $\mathrm{W} /(\mathrm{m} \cdot \mathrm{K})[13]$.

Thus, the most promising fillers for the VIPs are polydisperse powders consisting of amorphous porous particles of nano - and submicron size with a developed surface area and a network of voids of different size.

VIPs are widely used in refrigeration, but their use in construction is constrained by their high cost, which is formed, among the other factors, by the need for high vacuum and reliable sealing, which in turn involves the use of high-quality expensive materials. In this regard, the urgent task is to expand the range of the filler based on local materials and improve the technology of their production from the standpoint of economic.

\section{Initial materials}

Silica fume is formed as a by-product of the production of silicon, ferrochrome and other silicon alloys in electric arc furnaces as a result of cooling and filtration of furnace gases. Production of silicon alloys is energy-intensive, so such enterprises are usually located where there are sufficient electric power sources [14]. The structure and properties of amorphous silicon dioxide of various origins are well studied. This material has been used for the manufacture of ceramics, flame retardant compounds, plasticizers, as a filler in polymer composites. This material is most widely used in the manufacture of concretes and mortar. A number of countries, including Japan, Australia, France, Brazil, etc. have developed standards for the use of silicon dioxide in concretes and mortars. In the standards EN 13263-1:2005+A1:2009 Silica Fume for Concrete. Definitions, Requirements and Conformity Criteria and ASTM C1240 - 15 Standard Specification for Silica Fume Used in Cementitious Mixtures the basic requirements are presented for silica fume, as a component of cement materials $[15,16,17]$.

In contribution [9] on the base of data of measurement of a large set of samples it is shown that $\mathrm{SiO} 2$ spheres are not continuous, but consist of several densely packed spheres of a smaller size. It is suggested that small spheres may have a similar substructure. The result of this arrangement of spherical silicon dioxide particles will be the presence of tetrahedral and octahedral voids of various sizes: $\sim 40 \ldots 80 \mathrm{~nm}$ (large voids of the first order), $7 \ldots 14 \mathrm{~nm}$ (medium voids of the second order) and 2...4 $\mathrm{nm}$ (small voids of the third order) (classification of the authors [18]).

The structure of spherical particles of amorphous silica was studied by electron microscopy [19]. The authors have managed to show that relatively large ( $1000 \mathrm{~nm}$ ) spherical silica particles consist of smaller secondary particles with a diameter of $\sim 100 \mathrm{~nm}$, which in turn are composed of primary particles of $\sim 5 \ldots 10 \mathrm{~nm}$. In this case, large $\mathrm{SiO} 2$ particles can contain a central nucleus consisting of primary particles and surrounded by several rows of secondary particles.

According to H. Giesche [20], the size of subparticles of spherical particles of silicon dioxide is markedly less than $10 \mathrm{~nm}$ and can vary from sample to sample, being not always recorded by electron microscopy. In [21] a shell model for the structure of particles of amorphous silica obtained by the method of Stober - Fink - Bohn is shown. According to the authors, the $\mathrm{SiO} 2$ particle consists of a central nucleus composed of primary particles with a diameter of $5 \ldots 10 \mathrm{~nm}$, and the shells consisting of layers of secondary particles with a size of $20 \ldots 40 \mathrm{~nm}$, covered with layers of primary particles. The variants of the distribution of primary particles in the shells and the topology of the pore system arising at the same time are considered. Fractal and pore structures of silica particles are largely determined by the synthesis conditions and parameters of the medium in which it occurs [22].

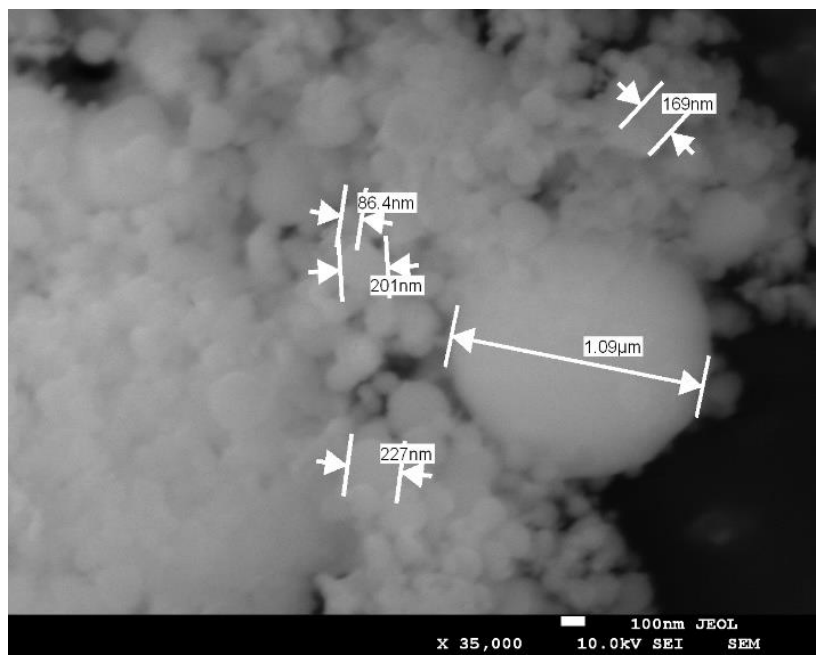

Figure 1. Microphotography of the particles of amorphous silica fume with an increase in $35000 X$.

\section{Methods}

Differential thermal analysis was conducted with the help of machine "STA-TG / DSK" of brand "STA 449 F 1 Jupiter". Curve lines DSK DTG and TG in the temperature interval of $25-1000^{\circ} \mathrm{C}$ were got for every kind of sample. Heating and cooling speed is. $10^{\circ} \mathrm{C} / \mathrm{min}$.; $\mathrm{X}$-ray diffraction analysis was performed by diffract meter "D 8 ADVANCE". Investigation of samples microstructure was made with the help of scanning electron microscope "JEOL JSM 700 1F". Thermal conductivity measurement of vacuum insulating panels worked by the authors out was performed by the 
stationary heat flux method applying thermal conductivity measurer "TCM MG 4 250".

We have conducted studies of the structure and properties of synthetic silica and micro silica - waste production. The study of the structure of the surface of the particles of silica fume was carried out by electron microscopy at a magnification of 50,000 and 35,000 times. On microphotography (Fig. 1) it can be seen that $\mathrm{SiO} 2$ spheres are not solid, but consist of several densely packed spheres of smaller size. Small spheres can have a similar substructure. The result of this arrangement of spherical particles of silicon dioxide is the presence of tetrahedral and octahedral voids of various sizes: $40 \ldots 80 \mathrm{~nm}, 7 \ldots 14 \mathrm{~nm}$ and $2 \ldots 4 \mathrm{~nm}$. The obtained results agree with the data of $[20,23,24]$ contributions, in which the shell model of the structure of amorphous silicon dioxide particles is discussed.

Investigation of synthetic silicon dioxide microphotography (Fig 2.) has shown that the size of the spherical particles of silicon dioxide is much smaller than $10 \mathrm{~nm}$ and can vary from sample to sample, being not always recorded by electron microscopy. This conclusion is consistent with the results of R. Ailer [25], who believes that the size of the sub-particles is less than 5 $\mathrm{nm}$. Agglomerates of amorphous silicon dioxide particles are observed in the form of diffuse sphere-like formations.

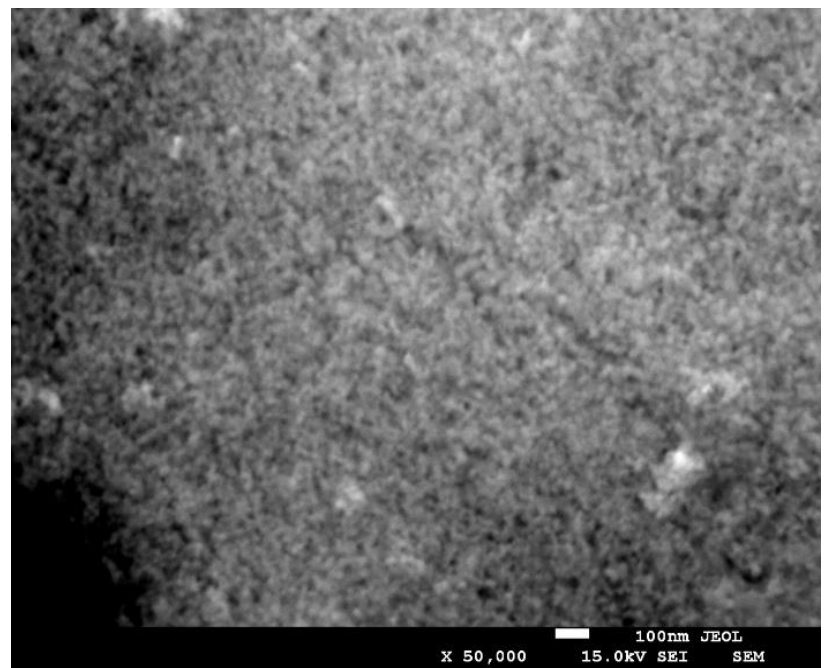

Figure 2. Microphotography of synthetic silica particles at 50000x magnification.

$\mathrm{X}$-ray diffraction analysis points that silica mainly consist of X-ray amorphous phase and blurry peak shows it in the area $18 \theta \ldots 30 \theta$. Crystal phase in the silica sample is actually absent and it is represented by $\beta$-quarts $(d=0,425 ; \quad d=0,335 ; \quad d=0,245 ; \quad d=0,154)$, carborundum $(d=0,251 ; d=0,154)$ and graphite $(d=0,335)$ fig. 3 .

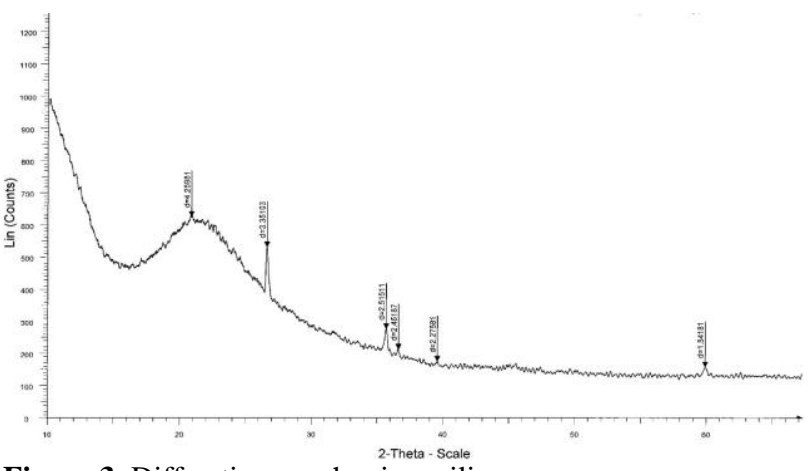

Figure 3. Diffraction graph micro silica.

Derivatives studies of micro silica showed the total weight loss of a sample being heated to $1000^{\circ} \mathrm{C}$ equal to $11,84 \%$, including to $220^{\circ} \mathrm{C}-8,5 \%$, from $220^{\circ} \mathrm{C}$ to $1000^{\circ} \mathrm{C}-3,1 \%$. \%. At the first stage there is weight loss because of removing free water from the sample. At the second stage there is smooth mass decreasing from the samples without visible physical and chemical transformations. The water presence $(\mathrm{t}=0-220 \mathrm{C})$ is marked by the curved lines TG and DTG. Thermal graphs are presented by figure 4 .

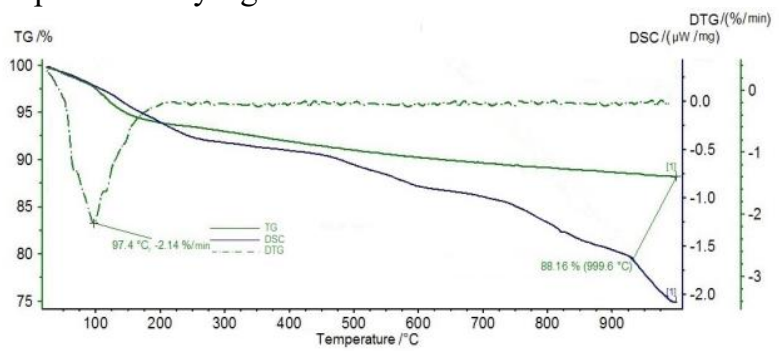

Figure 4. Thermal graphs micro silica.

Conducted research of COVELOS 35/01T synthetic silica and micro-silica of the aluminum plant confirm the existence of a developed pore structure of the particles of poly-disperse powder, which corresponds to a system with a large specific surface. The content of a large number of nanometer sized particles and pores make it possible to use these materials as a filler of vacuum insulation panels.

\section{Vacuuming composition and technology}

On the basis of amorphous micro- silica, synthetic silica and special additives, low-vacuum thermal insulation panels (VIPs) have been manufactured. The VIPs (Figure 5) have, as a rule, a flat parallelepiped form, but can be made in more complicated forms.

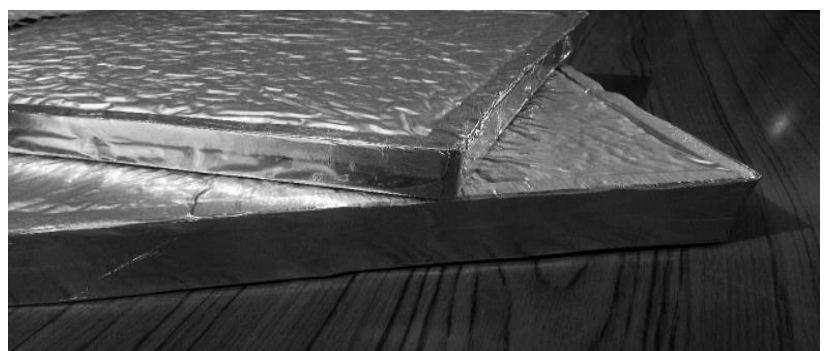

Figure 5. An external view of the vacuum insulated panels. 
The panels consist of an impermeable shell made of metalized polymer composite and the inner membrane layer made of fiberglass. The barrier frame made of gasfilled polymer was used as a shape forming element. The panels were filled with synthetic amorphous silicon dioxide and micro-silica - a waste of industry with addition and without addition of the infrared silencer for which titanium dioxide was chosen. Fillers were thoroughly mixed by sieving through a sieve and dried to a constant mass. The samples vacuuming were conducted at a pressure of $0.085 \mathrm{MPa}$. The hermiticity of the samples was provided by sealing the edges around the perimeter of the sample immediately after completion of the vacuuming process in a normal vacuum-packaging device.

\section{Thermal conductivity tests and discussion of results}

The VIPs thermal conductivity was determined at steadystate heat flow at medium temperatures plus $1{ }^{\circ} \mathrm{C}$ and plus $37.5^{\circ} \mathrm{C}$. The test results are shown in Figures 6 and 7 .

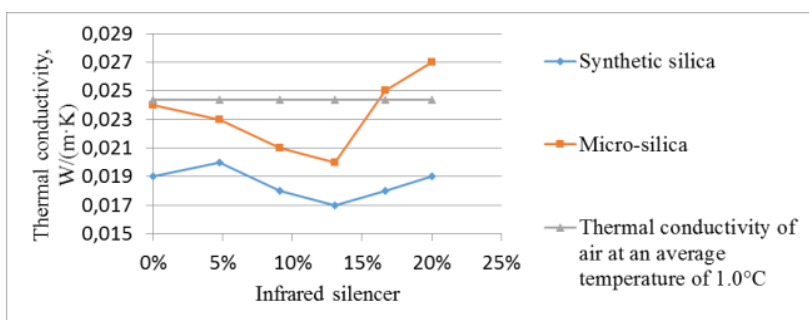

Figure 6. Thermal conductivity of the VIP samples at an average sample temperature of $1^{\circ} \mathrm{C}$.

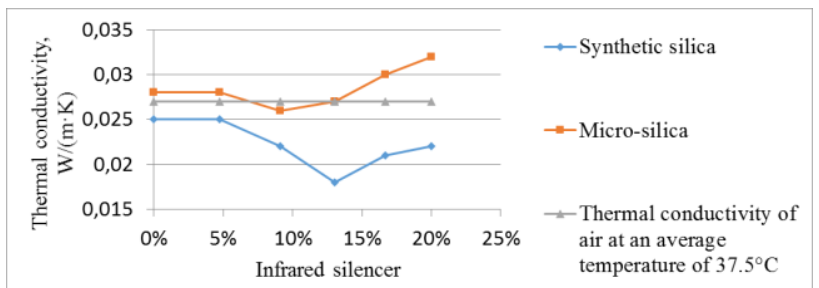

Figure 7. Thermal conductivity of the VIP samples at an average sample temperature of $37.5^{\circ} \mathrm{C}$.

The figures clearly show that thermal conductivity of the compositions based on synthetic silicon dioxide are lower than that of the micro - silica - waste of production. Lowering the average temperature of the samples during testing reduces thermal conductivity. This is mainly due to the fact that lowering the temperature reduces the thermal conductivity of the remaining air. The pressure of $0.085 \mathrm{MPa}$ in the manufacture of samples from the studied compositions of materials does not provide a significant reduction in thermal conductivity. The use of titanium dioxide becomes effective when its content is in the amount of $10 \ldots 15 \%$ compared to the weight of the filler. This effect is observed both for the samples of micro-silica and for synthetic silicon dioxide at elevated (plus $37.5^{\circ} \mathrm{C}$ ) and low (plus $1^{\circ} \mathrm{C}$ ) average temperatures. The thermal conductivity of the VIPs filled with micro-silica with the addition of titanium dioxide has a thermal conductivity lower than that of the air. Due to impermeability VIP do not change their heat insulating properties operating in high humid environment. The performed investigations of micro silica prove developed porous particle structure of polydespersed powders that corresponds the system with large specific surface. Disperse micro silica is aluminum plant industrial wastes can be used to produce low vacuum insulating panels VIP. Prospectively industry wastes applying can become the basis of high quality low net cost VIP manufacturing.

\section{References}

1. On approval of the strategy of innovative development of the Russian Federation for the period up to 20202011 The decree of the RF Government №2227-R

2. L. N. Danilevsky, AC 5 pp 114-7 (2006)

3. S. Oehler Münsterländer Hof, 10 Internationale Passivhaustagung pp 57-62 (2006)

4. N. Diefernbach Modernisierung von Zweifamilienhäusern, $\quad 10 \quad$ Internationale Passivhaustagung pp63-68 (2006)

5. R. A. Nazirov, N. S Novikov, and A. M. Zhzhonykh, ST 1 (25) pp 349-56 (2016)

6. G. N. Dul'nev and Y. P. Zarichnyak. Thermal conductivity of mixes and composite materials. Directory p 264 (1974)

7. G. N. Dulnev and V. V. Novikov, Transport process in inhomogeneous medium p 248 (1991)

8. A. Cherkashin, V. Pilipenko and L. Danilevsky AC. 1 p 219 (2011)

9. F. E. Aliev, N. K. Akhmedzhanova, V. F. Krivorotov and I. N. Holmanov et al. PSS 45 pp 60-67 (2003)

10. S. O. Gladkov, JTP 78 pp 12-15 (2008)

11. FRONT-VIP - Das Vakuum Isolationspaneel URL: http://www.vacu-isotec.de/vipfront.php?herkunft=baugewerbe

12. http://www.turna.si/Programi/Vakuumskoizolacijski paneliTURVAC.aspx

13. https://www.va-q-tec.com/de/

14. V. A. Shevchenko, N. A. Artemieva, L. A. Ivanova, V. P. Kiselev and G. V. Vasilovskaya, MPSE 1 p 2 (2015)

15. http://www.microsilicafume.eu/web/standards/10113 06087/list1187970081/f1.html

16. Guide for the Use of Silica Fume in Concrete (2012)

17. D. King, 37th Conference on OUR WORLD IN CONCR.\&STR. 74 (2012)

18. V. N. Bogomolov, L. S. Parfenyeva, A. V. Prokofiev and I. A. Smirnov, et al. PST 37 pp 3411-3418 (1995)

19. I. A. Karpov, E. N. Samarov, V. M. Masalov and S. I. Bozhko et al. 2005 PST 47 pp 334-338

20. H. Giesche JECS 14 pp189-204 (1994)

21. V. M. Masalov, N. S. Sukhinina and G. A. Emelchenko, CPST 2 pp 373-384 (2011)

22. K. D. Keefer and D. W. Schaefer, PRL 56 pp 23762379 (1986)

23. A. P. Philipse, CPS 12 pp 1174-1180 (1988)

24. G. H. Bogush and C.F. Zukoski, 1991 JCIS 142 pp 19-34

25. R. Iler, The Chemistry of Silica: Solubility, Polymerization, Colloid and Surface Properties and Biochemistry of Silica 866p (1979). 